\title{
Thyroid
}

\author{
Michel Polaka, Gabor Szinnaii ${ }^{b}$ Aurore Carréa and Mireille Castanet ${ }^{b}$ \\ apediatric Endocrinology and Gynecology, Hôpital Necker Enfants Malades, AP-HP, INSERM U845, \\ Université Paris Descartes, Paris, France \\ bPaediatric Endocrinology, University Children's Hospital, Basel, Switzerland
}

\begin{abstract}
The chapter aims at giving a representative choice of high-standing articles answering timely questions in the fields of thyroid physiology and disease covering areas as genetics, environmental factors, pharmacology, development and cancer. The concept of the year was the introduction of potassium channels as limiting factor of iodine accumulation in the murine thyroid by closing the gap between the sodium/iodide symporter (NIS) and the $\mathrm{Na}^{+}, \mathrm{K}^{+}$-ATPase by the presence of a constitutively active potassium channel. Time and future yearbooks will be able to estimate the clinical relevance of this report for understanding normal thyroid function and its disease.
\end{abstract}

\section{Mechanism of the year in thyroidology}

\section{Kcne2 deletion uncovers its crucial role in thyroid hormone biosynthesis}

Roepke TK, King EC, Reyna-Neyra A, Paroder M, Purtell K, Koba W, Fine E, Lerner DJ, Carrasco N, Abbott GW Greenberg Division of Cardiology, Department of Medicine and Department of Pharmacology, Weill Medical College of Cornell University, New York, N.Y., USA gwa2001@med.cornell.edu

Nat Med 2009;15:1186-1194

Background: Analogous to parietal cells and colonic crypt cells in the gastrointestinal tract, thyrocytes are non-excitable, polarized epithelial cells expressing ion transporters essential for the function of the thyroid gland. The thyroid hormones $(\mathrm{TH})$ triiodothyronine $\left(\mathrm{T}_{3}\right)$ and tetraiodothyronine (thyroxine, or $\left.\mathrm{T}_{4}\right)$ are critical for normal growth and development of the fetus and newborn as well as for regulation of metabolism in virtually all tissues at all ages. $\mathrm{I}^{-}$enters thyrocytes via the basolaterally located $\mathrm{Na}^{+} / \mathrm{I}^{-}$ symporter (NIS) and exits apically into the colloid, where it is covalently incorporated into thyroglobulin, the precursor of $\mathrm{T}_{3}$ and $\mathrm{T}_{4}$. NIS-mediated $\mathrm{I}^{-}$transport uses the downhill $\mathrm{Na}^{+}$gradient generated by the $\mathrm{Na}^{+}, \mathrm{K}^{+}$-ATPase at the basolateral membrane of the thyrocyte. The role of $\mathrm{K}^{+}$channels in the thyroid has not been described so far.

Results: Targeted disruption of Kcne2, a thyrocyte $\mathrm{K}^{+}$channel, in mice impaired thyroid iodide accumulation up to 8-fold, impaired maternal milk ejection, halved milk tetraiodothyronine $\left(\mathrm{T}_{4}\right)$ content and halved litter size. Kcne2-deficient mice had hypothyroidism, dwarfism, alopecia, goiter and cardiac abnormalities including hypertrophy, fibrosis, and reduced fractional shortening. The alopecia, dwarfism and cardiac abnormalities were alleviated by triiodothyronine $\left(\mathrm{T}_{3}\right)$ and $\mathrm{T}_{4}$ administration to pups, by supplementing dams with $\mathrm{T}_{4}$ before and after they gave birth or by feeding the pups exclusively from Kcne2+/+ dams; conversely, these symptoms were elicited in Kcne2+/+ pups by feeding exclusively from Kcne2-/- dams. Conclusion: The authors show that the potassium channel subunits KCNQ1 and KCNE2 form a thyroidstimulating hormone-stimulated, constitutively active, thyrocyte $\mathrm{K}^{+}$channel required for normal thyroid hormone biosynthesis. These data provide a new potential therapeutic target for thyroid disorders and raise the possibility of an endocrine component to previously identified KCNE2- and KCNQ1linked human cardiac arrhythmias.

For us, the concept of the year is the introduction of potassium channels in the scene of thyroid hormone biosynthesis. The authors identified the presence of a heterodimeric thyrocyte potassium channel, composed of the two subunits KCNQ1 and KCNE2. They showed that both subunits were expressed in human and murine thyrocytes, where they create a TSH-stimulated constitutively active potassium current. As supposed from their co-expression with the sodium/iodide symporter (NIS) at 
the basolateral membrane, deletion of Kcne2 resulted in an $\mathrm{I}^{-}$accumulation defect, the first step of thyroid hormone biosynthesis. Consequently, Kcne-l- mice were hypothyroid and developed goiter. Kcne-I- mice further had cardiac hypertrophy, and as published by the same group in a past paper, impaired ventricular repolarization.

Although the concept of closing the gap between the NIS and the $\mathrm{Na}^{+}, \mathrm{K}^{+}$-ATPase by the presence of a constitutively active potassium channel is new and innovative, human mutations in KCNE2 have been identified in patients with long QT syndrome (LQT subtype 6), but hypothyroidism was not reported in these patients so far. As subclinical hypothyroidism is also associated with prolonged QTC, a hallmark of loss-off-function mutations in KCNE2 and KCNQ1, thorough clinical description of thyroid function in patients with mutations is warranted to further support the hypothesis of a 'thyroid' component to some KCNE2- or KCNQ1-associated cardiac arrhythmias.

Whether further potassium channels are expressed in human thyrocytes and could be involved in human thyroid biosynthesis, remains unanswered for the moment.

\title{
Follow-up on a Yearbook 2009 paper (see Thyroid section, pp. 27-40) Protect the liver when using antithyroid drugs in children
}

\section{Dissimilar hepatotoxicity profiles of propylthiouracil and methimazole in children}

\author{
Rivkees SA, Szarfman A \\ Yale Pediatric Thyroid Center, Yale University School of Medicine, New Haven, Conn. and \\ Center for Drug Evaluation and Research, US Food and Drug Administration, Silver Spring, Md., USA \\ scott.rivkees@.yale.edu \\ J Clin Endocrinol Metab 2010, Epub ahead of print
}

Background: The antithyroid drugs propylthiouracil and methimazole are estimated to be used in more than 6,000 children and adolescents per year in the USA. Over the years that these medications have been used, reports of adverse events involving hepatotoxicity have appeared. The authors have already reported in a letter format the hepatotoxic effect of propylthiouracil in children and have pleaded not to use this drug anymore in pediatric Graves' disease [1]. However, there has not been a systematic and comparative evaluation of the adverse events associated with antithyroid drug use.

Objective: The authors' aim was to assess safety and hepatotoxicity profiles of propylthiouracil and methimazole by age in the US Food and Drug Administration's Adverse Event Reporting System (AERS).

Methods: They used a data mining algorithm to analyze more than 40 years of safety data in AERS. This algorithm allows ultimately calculating adjusted observed to expected ratios for every drug-adverse event combination in AERS, focusing on hepatotoxicity events.

Results: The algorithm identified higher-than-expected reporting of severe liver injury in pediatric patients treated with propylthiouracil but not with methimazole. Propylthiouracil had a high adjusted reporting ratio for severe liver injury (17; 90\% CI 11.5-24.1) in the group less than 17 years of age. The highest ratio values for methimazole were with mild liver injury in the group 61 years and older $(4.8(3.3-6.8)$ ), which consisted of cholestasis. Vasculitis was also observed for propylthiouracil in children and adolescents, reaching higher ratio values than hepatotoxicity signals.

Conclusions: Within the US Food and Drug Administration's AERS, the authors detected higher-thanexpected reporting of severe hepatotoxicity and vasculitis in children and adolescents with propylthiouracil but not with methimazole.

This detailed paper reinforces the preliminary message of last year's letter of the authors in the New England Journal of Medicine that propylthiouracil should not be used anymore in children with Graves' disease [1]. By use of a data mining algorithm of more than four decades of safety data of the FDA's AERS, they identified high reporting ratios for severe liver injury and vasculitis in the pediatric and adolescent age group for propiothiouracil vs. methimazole. The prevalence of severe liver failure was estimated to be approximately 1:2,000 children. This statement, derived from those FDA data, 
does not apply to adult patients [2]. In the treatment of pregnant women with active Graves' disease we are faced with a dilemma: propylthiouracil may appear as the drug of choice as methimazolerelated fetal malformations were reported in some studies but not in all [3-5]. A statement was issued to use propylthiouracil during organogenesis for the first 8 weeks of development and then to switch to methimazole [2]. However, the safety of both drugs in pregnant women as well as their potential teratogenic effects should therefore be under close scrutiny from now on and further data should be gathered before formal recommendations can be issued. This paper also highlighted the utility of systematic analyse adverse events reports in large databases using methods that decrease false-positive rates due to small numbers while preserving stable signals with a small number of reports.

\section{Clinical trials, new treatments \\ Pro and contra for in utero treatment of congenital hypothyroidism}

\section{Experience with intra-amniotic thyroxine treatment in non-immune fetal goitrous hypothyroidism in 12 cases}

Ribault V, Castanet M, Bertrand AM, Guibourdenche J, Vuillard E, Luton D, Polak M, the French Fetal Goiter Study Group

Pediatric Endocrinology, Université Paris Descartes, Necker Enfants Malades AP-HP, Paris, France

michel.polak@nck.aphp.fr

J Clin Endocrinol Metab 2009;94:3731-3739

Background: Non-immune fetal goitrous hypothyroidism is a rare condition that can induce obstetrical and/or neonatal complications and neurodevelopmental impairments such as those still seen in some patients with congenital hypothyroidism. Prenatal treatment to prevent these adverse outcomes is appealing, but experience is limited and the risk-to-benefit ratio controversial.

Objective: The authors wished to evaluate the feasibility, safety, and effectiveness of intrauterine L-thyroxine treatment, using intra-amniotic injections in a large cohort with non-immune fetal goitrous hypothyroidism.

Methods: This study was a retrospective study of 12 prenatally treated fetuses diagnosed between 1991 and 2005 in France. During pregnancy, goiter size and thyroid hormone levels were compared before and after prenatal treatment. At birth, clinical, laboratory, and ultrasound data were evaluated.

Results: The practice of the prenatal treatment was found to vary widely in terms of L-thyroxine dosage (200-800 $\mu \mathrm{g} /$ injection), number of injections (1-6), and frequency (every 1-4 weeks). Fortunately, no adverse events were recorded. During pregnancy, thyroid size decreased in 8 of 9 cases and amnioticfluid TSH levels decreased in the 6 investigated cases, returning to normal in 4. However, at birth, all babies had hypothyroidism, indicating that intra-amniotic TSH levels did not reliably reflect fetal thyroid function.

Conclusion: The authors confirmed the feasibility and safety of intra-amniotic L-thyroxine treatment for non-immune fetal goitrous hypothyroidism. They also could show that amniocentesis seemed inadequate for monitoring fetal thyroid function in comparison with fetal blood sampling. However, further studies are needed to determine the optimal management of this disorder. 


\section{Non-immune goiter and hypothyroidism in a 19-week fetus: a plea for conservative treatment}

Stoppa-Vaucher S, Francoeur D, Grignon A, Alos N, Pohlenz J, Hermanns P, Van Vliet G, Deladoëy J

Endocrinology Service and Research Center and Department of Pediatrics, CHU Sainte-Justine and

Université de Montréal, Montréal, Que., Canada

Johnny.deladoey@umontreal.ca

J Pediatr 2010;156:1026-1029

Hypothyroidism was documented by cordocentesis at 19 weeks in a fetus with non-immune goiter. Intra-amniotic thyroxine was injected at 25 weeks when amniotic fluid volume increased. Psychomotor outcome was normal. The authors argue that intra-amniotic thyroxine should not be used to treat the hypothyroidism but only to correct the development of polyhydramnios.

The diagnosis of fetal thyroid function disturbances is now possible in utero [6-8]. The feasibility of intrauterine treatment of fetuses affected by hypothyroidism has been shown. The incompletely solved question is when should we apply those new tools? While the first paper reports a multicenter retrospective experience with in utero treatment, the second paper reports a case with a review of the literature. Fetal goiter size decreased in 8 of 9 investigated cases and intra-amniotic TSH levels decreased. Some authors propose to use intra-amniotic thyroxine treatment only in case of fetal goiter that may impede vaginal delivery or result in the development of polyhydramnios, others would favor an approach using research protocols to target fetal hypothyroidism treatment itself $[7,8]$. The authors rightly conclude that 'to determine the indications and optimal modalities of the prenatal treatment of non-immune fetal goitrous hypothyroidism, larger and well-designed studies are needed and would be best conducted via international cooperation of multidisciplinary medical teams'.

\section{Important for clinical practice Everlasting questions on congenital hypothyrodism screening}

\section{Newborn screening results in children with central hypothyroidism}

Nebesio TD, McKenna MP, Nabhan ZM, Eugster EA

Indiana University School of Medicine, Department of Pediatrics, Riley Hospital for Children, Indianapolis, Ind., USA tdnebesi@iupui.edu

J Pediatr 2010;156:990-993

Background: The authors wished to investigate newborn screening results in children with congenital hypopituitarism, due to central hypothyroidism in a screening program that uses $\mathrm{T}_{4}$ determination. They also wished whether there were differences between children who had abnormal results and children with normal newborn screening results, all with central hypothyroidism.

Methods: Medical records of children with central hypothyroidism observed in their pediatric endocrinology clinics from 1990 to 2006 were reviewed.

Results: 42 subjects ( 22 boys) were identified. 8 children (19\%) had a low total thyroxine level $(<5.0 \mu \mathrm{g} /$ dl) on the newborn screening test. The average total thyroxine level in the remaining 34 subjects was $9.8 \pm 3.4 \mu \mathrm{g} / \mathrm{dl}$. Thyrotropin levels were within the reference range in all children, as expected but not adapted for those with low $\mathrm{T}_{4}$ levels. No differences were found in the two groups for birth history, jaundice $(53 \%$ overall), hypoglycemia (36\% overall), or micropenis $(43 \%$ of boys). $57 \%$ of children had septo-optic dysplasia, and $98 \%$ had multiple pituitary hormone deficiencies. Children with an abnormal newborn screening results were initially examined by a pediatric endocrinologist at an average age of $4.6 \pm 5.0$ months, and children with normal newborn screening results were initially examined at an average age of $16.9 \pm 26.7$ months $(p=0.037)$.

Conclusions: Most children with congenital central hypothyroidism have normal thyroid function at birth. Normal newborn screening results can be falsely reassuring and may contribute to a delay in diagnosis of hypopituitarism despite classic clinical features. 


\title{
A 7-year experience with low blood TSH cut-off levels for neonatal screening reveals an unsuspected frequency of congenital hypothyroidism
}

Corbetta C, Weber G, Cortinovis F, Calebiro D, Passoni A, Vigone MC, Beck-Peccoz P, Chiumello G, Persani L

Laboratory for Neonatal Screening, Buzzi Children Hospital, Milan, Italy

luca.persani@unimi.it

Clin Endocrinol (Oxf) 2009;71:739-745

Background: The guidelines of the National Academy of Clinical Biochemistry [www.nabc.org] advocated the use of low bloodspot TSH (b-TSH) threshold for newborn screening of congenital hypothyroidism $(\mathrm{CH})[9]$.

Objective: The authors wished to determine the impact on $\mathrm{CH}$ epidemiology and classification generated by the introduction of lower b-TSH cut-off than the on they previously used.

Methods: Retrospective study of 629,042 newborns screened with b-TSH cut-offs of 12 (years 19992002) or $10 \mathrm{mU} / \mathrm{l}$ (2003-2005). Results were compared with those virtually obtained with the previous cut-off $(20 \mathrm{mU} / \mathrm{l})$. Clinical re-evaluation after $\mathrm{L}_{-} \mathrm{T}_{4}$ withdrawal of a representative group of $140 \mathrm{CH}$ children at 3-5 years was also performed.

Results: Low b-TSH cut-offs allowed the detection of 435 newborns with confirmed $\mathrm{CH}$ (incidence $1: 1,446) .45 \%$ of $\mathrm{CH}$ infants, including $12 / 141$ dysgenesis, would have been missed using the $20 \mathrm{mU} / \mathrm{l}$ cut-off. In contrast to current classification, 32\% CH newborns had thyroid dysgenesis and $68 \%$ had a gland in situ (GIS). Premature birth was present in $20 \%$ of cases being associated with a 3- to 5 -fold increased risk of GIS CH. Re-evaluation at 3-5 years showed a permanent thyroid dysfunction in $78 \%$ of $59 \mathrm{CH}$ toddlers with GIS.

Conclusions: The use of low b-TSH cut-off allowed the detection of an unsuspected number of children with neonatal hypothyroidism, evolving in mild permanent thyroid dysfunction later in life. The incidence of $\mathrm{CH}$ in this Italian population appears to be double than previously thought with a clear-cut prevalence of functional defects over dysgenetic ones.

\section{Difficulties in selecting an appropriate neonatal thyroid-stimulating hormone screening threshold}

\author{
Korada SM, Pearce M, Ward Platt MP, Avis E, Turner S, Wastell H, Cheetham T \\ Institute of Human Genetics, Newcastle University, Department of Paediatrics, Royal Victoria Infirmary, \\ Newcastle upon Tyne, UK \\ tim.cheetham@nuth.nhs.uk \\ Arch Dis Child 2010;95:169-173
}

Background: The UK Newborn Screening Programme Centre recommends that a blood spot thyroid-stimulating hormone (TSH) cut-off of $10 \mathrm{mU} / \mathrm{l}$ is used to detect congenital hypothyroidism (CHT). As the value used varies from 5 to $10 \mathrm{mU} / \mathrm{l}$, we examined the implications of altering this threshold.

Methods: The authors' regional blood spot TSH cut-off is $6 \mathrm{mU} / \mathrm{l}$. Positive or suspected cases were defined as a TSH $>6 \mathrm{mU} / \mathrm{l}$ throughout the study period (1 April 2005 to 1 March 2007). All term infants (>35 weeks) whose first TSH was $6-20 \mathrm{mU} / \mathrm{l}$ had a second TSH measured. The biochemical details of infants with a TSH between 6.1 and $10.0 \mathrm{mU} / \mathrm{l}$ and then $>6 \mathrm{mU} / \mathrm{l}$ on second sampling were sent to pediatric endocrinologists to determine approaches to management.

Results: 148 of 65,446 infants $(0.23 \%)$ had a first blood spot TSH $>6.0 \mathrm{mU} / 1.120$ were term infants with 67 of these $(0.1 \%$ of all infants tested) having a TSH between 6.1 and $10.0 \mathrm{mU} / \mathrm{l}$ and 53 a TSH $>10.0 \mathrm{mU} / \mathrm{l}$. Of the 67 term infants with a TSH between 6.1 and $10.0 \mathrm{mU} / \mathrm{l}$ on initial testing, 4 continued to have a TSH $>6 \mathrm{mU} / \mathrm{l}$. One with a TSH $>10 \mathrm{mU} / \mathrm{l}$ and 1 infant with a TSH $<10 \mathrm{mU} / \mathrm{l}$ on the second blood spot have been diagnosed with CHT. The survey of endocrinologists highlighted significant differences in practice.

Conclusions: A reduced threshold of $6 \mathrm{mU} / \mathrm{l}$ will increase the number of false positive term infants by $126 \%$, but abnormalities of thyroid function requiring treatment will be detected. The authors suspected but did not confirm that the additional expense involved in setting a lower threshold is justified. 


\section{Neonatal hyperthyrotropinemia: population characteristics, diagnosis, management and outcome after cessation of therapy}

Zung A, Tenenbaum-Rakover Y, Barkan S, Hanukoglu A, Hershkovitz E, Pinhas-Hamiel O, Bistritzer T, Zadik Z

Pediatric Endocrinology Unit, Kaplan Medical Center, Rehovot, Israel

amzung2@bezeqint.net

Clin Endocrinol (Oxf) 2010;72:264-271

Background: Neonatal hyperthyrotropinemia (HT), defined by elevated TSH and normal $\mathrm{T}_{4}$, is either transient or persistent.

objective: The authors assessed perinatal parameters and diagnostic measures that may distinguish between transient and persistent HT, compared with congenital hypothyroidism $(\mathrm{CH})$. They aimed to recommend optimal treatment in these forms of neonatal thyroid impairment.

Methods: A multi-centre, retrospective study was conducted in six pediatric endocrinology units. 43 HT patients and $83 \mathrm{CH}$ patients were included in the study. The authors evaluated differences in birth weight (BW), gestational age (GA), modes of diagnosis, screening and confirmatory $\mathrm{T}_{4}$ and TSH levels, thyroid imaging results and optimal thyroxine doses between $\mathrm{HT}$ and $\mathrm{CH}$ and between the two forms of HT.

Results: Newborns with HT had lower BW and GA than those with $\mathrm{CH}$. Transient $(\mathrm{n}=18)$ and persistent HT $(n=25)$ patients were indistinguishable by most parameters, but those with persistent HT had a higher prevalence of abnormal thyroid imaging ( 69 vs. $8 \% ; p=0.005)$. During treatment, 79 and $55 \%$ of transient and persistent HT patients respectively experienced elevated levels of free $\mathrm{T}_{4}$. Although most HT patients were re-evaluated after 2.5 years, 6 transient HT patients stopped therapy and showed full recovery within the first year of life.

Conclusion: The authors recommended obtaining thyroid imaging to distinguish between the two forms of HT. They also advised that early cessation of therapy in transient HT can prevent iatrogenic hyperthyroidism in these patients.

These four articles targeted questions related to screening of congenital hypothyroidism and extend our current knowledge.

The first paper analyzed the sensitivity of combined $\mathrm{T}_{4} / \mathrm{TSH}$ bloodspot screening for detection of neonatal central hypothyroidism. Proponents of a $\mathrm{T}_{4}$ screening claim that this would identify central hypothyroidism. Only $8(19 \%)$ of a retrospective single-center cohort of 42 children with central hypothyroidism were detected by decreased $T_{4}$ values in the newborn screening. The diagnosis of central hypothyroidism was based on classic clinical and laboratory features rather than dynamic testing. This rate of normal newborn screening results of $T_{4}$ in central hypothyroidism is much higher than stated in previous reports $[10,11]$. This article reminds us that the possibility of late congenital hypothyroidism diagnosis should be on our mind despite normal screening results and that the clinical skills to suspect such a diagnosis should still be taught.

Two papers questioned the threshold of the bloodspot TSH levels for the neonatal screening of congenital hypothyroidism. As expected, more cases were found when lowering the bloodspot TSH cutoff value to 12, 10 or $6 \mathrm{mU} / \mathrm{l}$. Of interest, several cases of thyroid dysgenesis were found in both cohorts. On the other hand, many patients with glands in situ were identified with a high incidence of permanent thyroid dysfunction at follow-up.

The worrisome question is: What happened to the children with such intermediate levels of TSH on screening at the time when the threshold was higher: were they picked up at a later age with mild hypothyroidism, should we be worried for their cognitive development?

The fourth paper raised the question of the follow-up of children with neonatal hyperthyrotropinemia (HT) based on a retrospective analysis. Patients with permanent HT had a higher prevalence of abnormal thyroid imaging but were indistinguishable by other parameters from patients with transient HT. Of interest, the authors demonstrated a significant incidence of iatrogenic hyperthyroidism due to treatment. They draw our attention to this peculiar group of patients in whom a targeted dose of thyroxine and a close follow-up is necessary. 


\section{Medullary thyroid cancer: management guidelines of the American Thyroid Association}

American Thyroid Association Guidelines Task Force, Kloos RT, Eng C, Evans DB, Francis GL, Gagel RF, Gharib H, Moley JF, Pacini F, Ringel MD, Schlumberger M, Wells SA Jr

Department of Internal Medicine, Division of Endocrinology, Diabetes and Metabolism, The Ohio State University, The Arthur G. James Cancer Hospital, Columbus, Ohio, USA

richard.kloos@osumc.ed

Thyroid 2009;19:565-612

Background: Inherited and sporadic medullary thyroid cancer (MTC) is an uncommon and challenging malignancy. The American Thyroid Association (ATA) chose to create specific MTC Clinical Guidelines that would bring together and update the diverse MTC literature and combine it with evidence-based medicine and the knowledge and experience of a panel of expert clinicians.

Methods: Relevant articles were identified using a systematic PubMed search and supplemented with additional published materials. Evidence-based recommendations were created and then categorized using criteria adapted from the United States Preventive Services Task Force, Agency for Healthcare Research and Quality.

Results: The authors addressed initial diagnosis and therapy of preclinical disease (including RET oncogene testing and the timing of prophylactic thyroidectomy), initial diagnosis and therapy of clinically apparent disease (including preoperative testing and imaging, extent of surgery, and handling of devascularized parathyroid glands), initial evaluation and treatment of postoperative patients (including the role of completion thyroidectomy), management of persistent or recurrent MTC (including the role of tumor marker doubling times, and treatment of patients with distant metastases and hormonally active metastases), long-term follow-up and management (including the frequency of follow-up and imaging), and directions for future research.

Conclusion: 122 evidence-based recommendations were created to assist in the clinical care of MTC patients and to share what the authors believed to be current, rational, and optimal medical practice.

This paper updates the 2001 consensus guidelines for treatment of MEN2 and MTC patients based on the important advances of knowledge over the last 10 years in the application of 'codon-specific prophylactic thyroidectomy' which is the gold standard for kindred in MEN2 families. Recommendations are stratified according to more experience with clinical outcome of patients with specific RET mutations, especially with rare ones. The management guidelines further propose a large series of recommendations dealing with diagnosis, surgical approach and long-term follow-up for medullary thyroid carcinoma, as well as for MEN2-associated pheochromocytoma and hyperparathyroidism. The 122 recommendations give answers based on the current state of the art to all clinical situations encountered in the care of affected families, from controversies concerning counseling of prenatal testing to management of persistent or recurrent metastatic disease.

While the paper shows the way for the optimal management, the application of these recommendations in clinical life, especially also by pediatric endocrinologists, will need to be evaluated in the future.

\section{Second malignant neoplasms in survivors of pediatric Hodgkin's lymphoma treated with low-dose radiation and chemotherapy}

O'Brien MM, Donaldson SS, Balise RR, Whittemore AS, Link MP

Department of Pediatric Hematology/Oncology, Cincinnati Children's Hospital Medical Center, Cincinnati, Ohio, USA maureen.obrien@cchmc.org

J Clin Oncol 2010;28:1232-1239

Background: Survivors of childhood Hodgkin's lymphoma (HL) are at risk for second malignant neoplasms (SMNs). It is theorized that this risk may be attenuated in patients treated with lower doses of 
radiation. We report the first long-term outcomes of a cohort of pediatric survivors of HL treated with chemotherapy and low-dose radiation.

Methods: Pediatric patients with HL $(\mathrm{n}=112)$ treated at Stanford from 1970 to 1990 on two combined modality treatment protocols were identified. Treatment included six cycles of chemotherapy with 15-25.5 Gy involved-field radiation with optional 10-Gy boosts to bulky sites. Follow-up through September 1, 2007, was obtained from retrospective chart review and patient questionnaires.

Results: 110 children completed HL therapy; median follow-up was 20.6 years. 18 patients developed one or more SMNs, including 4 leukemias, 5 thyroid carcinomas, 6 breast carcinomas, and 4 sarcomas. Cumulative incidence of first SMN was $17 \%(95 \%$ CI 10.5-26.7) at 20 years after HL diagnosis. The standard incidence ratio for any SMN was 22.9 (95\% CI 14.2-35) with an absolute excess risk of 93.7 cases per 10,000 person-years. All 4 secondary leukemias were fatal. For those with second solid tumors, the mean $( \pm \mathrm{SE}) 5$-year disease-free and overall survival were $76 \pm 12 \%$ and $85 \pm 10 \%$ with median follow-up 5 years from SMN diagnosis.

Conclusion: Despite treatment with low-dose radiation, children treated for HL remain at significant risk for SMN. Sarcomas, breast and thyroid carcinomas occurred with similar frequency and latency as found in studies of children with HL who received high-dose radiation.

O'Brien et al. show that low-dose irradiation of the thyroid in the context of pediatric Hodgkin's lymphoma results in a significant risk for thyroid carcinoma on follow-up. Although reflecting the consequence of past therapies, the authors expect a reduction of second malignant neoplasms such as breast cancer by further dose reduction and shielding procedures, but not for thyroid cancer. They advocate an 'aggressive surveillance' of these patients. The most accurate follow-up remains to be determined but it should most probably include yearly thyroid ultrasound.

As decreasing intensity of clinical follow-up of these patients often coincides with the transition from the pediatric oncologist and endocrinologist to the adult endocrinologist or general practitioner, the risk of second malignant neoplasias needs to be well communicated to the patient and the responsible physicians.

\title{
Long-term outcome in 215 children and adolescents with papillary thyroid cancer treated during 1940 through 2008
}

\author{
Hay ID, Gonzalez-Losada T, Reinalda MS, Honetschlager JA, Richards ML, Thompson GB \\ Division of Endocrinology and Internal Medicine, Mayo Clinic, Rochester, Minn., USA \\ hay.ian@mayo.edu
}

World J Surg. 2010;34:1192-1202

Background: Controversy exists regarding the aggressiveness of initial therapy in childhood papillary thyroid cancer (PTC). Few studies with long-term outcome exist and second primary malignancies have rarely been analyzed.

Methods: The authors studied 215 PTC patients younger than 21 years of age who were managed during 1940 through 2008. The patients were aged 3-20 years (median 16 years); median follow-up was 29 years. Recurrence and mortality details were taken from a computerized database.

Results: Median primary tumor size was $2.2 \mathrm{~cm}$. Six percent had distant metastases at presentation, $5 \%$ had incomplete tumor resection, $86 \%$ had nodes removed at initial surgery, and $78 \%$ had nodal metastases. After complete surgical resection, PTC recurred in $32 \%$ by 40 years. At 20 years, the recurrence rates at local, regional, and distant sites were 7, 21, and 5\%, respectively. During 1940-1969, local and regional recurrence rates after unilateral lobectomy (UL) were significantly $(\mathrm{p}<0.001)$ higher than after bilateral lobar resection (BLR). During 1950-2008, radioiodine remnant ablation (RRA) was administered within 18 months to $32 \%$; it did not diminish the 25 -year regional recurrence rate of $16 \%$ seen after BLR alone $(\mathrm{p}=0.86)$. Only 2 fatal events from PTC occurred at 28 and 30 years, for a causespecific mortality at 40 years of only $2 \%$. All-causes mortality rates did not exceed expectation through 20 years, but from 30 through 50 years, the number of deaths was significantly $(\mathrm{p}<0.001)$ higher than predicted. 15 of 22 deaths (68\%) resulted from non-thyroid malignancy.

Conclusion: Survival from childhood PTC should be expected, but later death from non-thyroid malignancy is disconcerting. $73 \%$ of those who died from non-thyroid malignancy had received postoperative therapeutic irradiation. 
In general, childhood PTC certainly has an excellent prognosis. Owing to an extensively long followup period of 40 years, Han et al. revealed an unexpectedly high mortality of patients treated for PTC below age 21 years due to second primary tumors of solid organs other than thyroid between 30 and 50 years of age. The majority $(73 \%)$ of these patients received postoperative therapeutic irradiation. Further, they showed that radioiodine remnant ablation did not diminish the 25 -year regional recurrence rate. This result was surprising and suggests that irradiation of this patient group should be avoided as far as possible. However, in this large, single institutional cohort, the differences between survival after surgery alone and after surgery plus RRA to the endpoints of local recurrence, neck nodal metastases, locoregional recurrence, distant metastases, and all-sites recurrence were shown to be statistically insignificant. Lacking a more thorough description of the patients treated with radioiodine and the selection process of those receiving such a procedure may have induced such results. The current use of radioiodine has been validated in others studies [12].

\section{New genetic studies for known and unknown genes}

\section{NKX2-1 mutations leading to surfactant protein promoter dysregulation cause interstitial lung disease in 'brain-lung-thyroid syndrome'}

Guillot $\mathrm{L}^{\mathrm{a}}$, Carré $\mathrm{A}^{\mathrm{a}}$, Szinnai $\mathrm{G}^{\mathrm{b}}$, Castanet $\mathrm{M}^{\mathrm{b}}$, Tron $\mathrm{E}^{\mathrm{b}}$, Jaubert $\mathrm{F}^{\mathrm{b}}$, Broutin $\mathrm{I}^{\mathrm{b}}$, Counil $\mathrm{F}^{\mathrm{b}}$, Feldmann $\mathrm{D}^{\mathrm{b}}$, Clement $\mathrm{A}^{\mathrm{b}}$, Polak Ma $M^{a}$ Epaud $R^{a}$

aINSERM UMR 938 and U845, Université Paris 6 and Université Paris Descartes and

bHôpital Trousseau and Necker Enfants Malades, Paris, France

michel.polak@nck.aphp.fr, ralph.epaud@trs.aphp.fr

Hum Mutat 2010;31:E1146-1162

Background: NKX2-1 (NK2 homeobox 1) is a critical regulator of transcription for the surfactant protein (SP)-B and -C genes (SFTPB and SFTPC, respectively).

Results: The authors identified and functionally characterized two new de novo NKX2-1 mutations c.493C > T (p.R165W) and c.786_787del2 (p.L263fs) in infants with closely similar severe interstitial lung disease (ILD), hypotonia, and congenital hypothyroidism. Functional analyses using A549 and HeLa cells revealed that NKX2-1-p.L263fs induced neither SFTPB nor SFTPC promoter activation and had a dominant negative effect on wild-type (WT) NKX2-1. In contrast, NKX2-1-p.R165W activated SFTPC, to a significantly greater extent than did WTNKX2-1, while SFTPB activation was only significantly reduced in HeLa cells. In accordance with their in vitro data, the authors found decreased amounts of SP-B and SP-C by Western blot in bronchoalveolar lavage fluid (patient with p.L263fs) and features of altered surfactant protein metabolism on lung histology (patient with NKX2-1-p. R165W).

Conclusion: ILD in patients with NKX2-1 mutations was associated with altered surfactant protein metabolism, and both gain and loss of function of the mutated NKX2-1 genes on surfactant protein promoters were associated with ILD in 'brain-lung-thyroid syndrome'. In contrast, in the thyroid both mutations led to loss of function.

Since the first descriptions of NKX2-1/TITF1 (NK2 homeobox 1/thyroid transcription factor 1) mutations leading to the clinical triad of congenital hypothyroidism, surfactant deficiency syndrome and benign hereditary chorea, also called 'brain-lung-thyroid syndrome' [13], the lung disease remained the 'white spot' of the disease. The data presented showed for the first time in vivo and in vitro that patients with NKX2-1 mutations with lung disease suffered from disturbed surfactant protein metabolism. This study further highlighted the variability of the transactivation capacity of mutated NKX21 , as both gain and loss of function on surfactant protein promoters were associated with interstitial lung disease, probably due to altered interaction of transcriptional activators and inhibitors. The data of the paper are consistent with experimental evidence documenting the importance of NKX2-1 in pulmonary surfactant metabolism. For the neonatologists and pneumologists it is of importance to search for NKX2-1 mutations in newborns presenting neonatal surfactant deficiency syndrome with mild hyperthyrotropinemia. Whether isolated lung disease due to NKX2-1 mutations in analogy with 


\section{Compound heterozygosity for a novel hemizygous missense mutation and a partial deletion affecting the catalytic core of the $\mathrm{H}_{2} \mathrm{O}_{2}$-generating enzyme DUOX2 associated with transient congenital hypothyroidism}

Hoste C, Rigutto S, Van Vliet G, Miot F, De Deken X

IRIBHM, Université Libre de Bruxelles, Campus Erasme, Brussels, Belgium

xdedeken@ulb.ac.be

Hum Mutat 2010;31:E1304-1319

Background: Dual oxidases (DUOX) 1 and 2 are components of the thyroid $\mathrm{H}_{2} \mathrm{O}_{2}$-generating system. $\mathrm{H}_{2} \mathrm{O}_{2}$ is used by thyroperoxidase to oxidize iodide for thyroid hormonogenesis. Mutations in the DUOX2 gene have been described in transient and permanent congenital thyroid dyshormonogenesis. Results: The authors report here a novel genetic defect causing congenital hypothyroidism in a FrenchCanadian patient. At neonatal screening, the patient had high TSH and low total $\mathrm{T}_{4}$ levels. ${ }^{99 \mathrm{~m} T \mathrm{~T}}$ scan showed a normally shaped orthotopic but mildly enlarged thyroid gland, suggesting dyshormonogenesis. Thyroxine treatment was given from 1 month to 17 years, after which it was stopped for re-evaluation and the patient remained euthyroid. The transient congenital hypothyroidism phenotype prompted the authors to screen for mutations in DUOX2 and DUOXA2 genes using the PCR-amplified direct sequencing method. They found complete inactivation of DUOX2 caused by a partial genomic deletion of one allele inherited from the mother associated with a paternally inherited missense mutation (c.4552G>A, p.Gly1518Ser). The deleted fragment encompasses the entire COOH-terminal end which is responsible for the NADPH-oxidase activity. The Gly1518Ser DUOX2 protein is expressed at the cell surface of transfected cells albeit at low level, but it is non-functional.

Conclusion: This study provides further evidence that the permanent or transient nature of congenital hypothyroidism is not directly related to the number of inactivated DUOX2 alleles, suggesting the existence of other pathophysiological factors.

The biochemical requirement of $\mathrm{H}_{2} \mathrm{O}_{2}$ for thyroid hormone synthesis has been known for decades. Two homologous proteins (dual oxidase 1 and 2) containing functional domains of NADPH oxidoreductases were identified in thyroid follicular cells. Mutations in the dual oxidase 2 gene (DUOX2, formerly THOX2) gene have been shown to cause transient and permanent dyshormonogenetic congenital hypothyroidism. So far, transient forms were associated with heterozygous mutations, and permanent forms with homozygous mutations in DUOX2 [14].

This interesting case, reporting a patient with transient congenital hypothyroidism and compound heterozygosity for a novel hemizygous missense mutation and a partial deletion of DUOX2, raises the question whether transiency and permanency of congenital hypothyroidism relate exclusively to monoallelic or biallelic inactivation of the gene or whether disease phenotype is dependent on residual function of the mutated DUOX2 protein. Further work on more patients affected with DUOX2 gene mutations is awaited to clarify this fundamental question.

\section{Screening chromosomal aberrations by array comparative genomic hybridization in $\mathbf{8 0}$ patients with congenital hypothyroidism and thyroid dysgenesis}

Thorwarth A, Mueller I, Biebermann H, Ropers HH, Grueters A, Krude H, Ullmann R

Institute for Experimental Pediatric Endocrinology, Charité University Medicine and Max Planck Institute for

Molecular Genetics, Berlin, Germany

heiko.krude@charite.de, ullmann@molgen.mpg.de

J Clin Endocrinol Metab 2010, Epub ahead of print

Background: A spectrum of defective thyroid morphology, termed thyroid dysgenesis (TD), represents $80 \%$ of permanent congenital hypothyroidism cases. Although several candidate genes have been implicated in thyroid development, comprehensive screens failed to detect mutation carriers in a significant number of patients with non-syndromic TD. 
Objective: Due to the sporadic occurrence of TD, the authors aimed at assessing de novo chromosomal rearrangements which conceivably represents one of the molecular mechanisms participating in its etiology.

Methods: The introduction of array comparative genomic hybridization (CGH) has provided the ability to map DNA copy number variations (CNVs) genome-wide with high resolution. The authors performed an array CGH screen of 80 TD patients to determine the role of CNVs in the etiology of the disease.

Results: They identified novel CNVs that have not been described as frequent variations in the healthy population in $8.75 \%$ of all patients. These CNVs exclusively affected patients with athyreosis or thyroid hypoplasia and were non-recurrent, and the regions flanking the CNVs were not enriched for segmental duplications.

Conclusions: The high rate of chromosomal changes in TD argues for an involvement of CNVs in the etiology of this disease. Yet the lack of recurrent aberrations suggests that the genetic causes of TD are heterogenous and not restricted to specific genomic hot spots. Thus, future studies may have to shift the focus from singling out specific genes to the identification of deregulated pathways as the underlying cause of the disease. As in others types of endocrine disorders as well as in others fields of medicine, the study of DNA CNVs may turned out to be productive to find the etiologies of TD [15].

New methods for microarray-based genotyping of single nucleotide polymorphism (SNP) markers as well as parallel methods assessing rare and common DNA deletions or duplications across the genome as copy number variants (CNVs) permitted breakthrough identification of susceptibility genes for nonmendelian diseases $[16,17]$. A recent large-scale analysis of CNVs in eight common human diseases puts the contribution of CNVs in comparison with SNP-based studies for detection of new genetic hot spots into perspective [18; also see Science and Medicine section by Z. Hochberg and J.-C. Carel, pp. 219-236]. The study of Thorwarth et al. searched for the first time for common chromosomal changes in a representative cohort of patients with thyroid dysgenesis. Their results add to current knowledge the fact that a high rate of chromosomal changes was found in these patients. However, the absence of recurrent CNVs further supports the notion that thyroid dysgenesis is rather a heterogenous disease than a monogenetic one. Future approaches aiming to decipher the genetic basis of thyroid dysgenesis should consider this new piece of knowledge.

\section{Environment}

\section{lodine supplementation improves cognition in mildly iodine-deficient children}

Gordon RC, Rose MC, Skeaff SA, Gray AR, Morgan KM, Ruffman T

Department of Human Nutrition, University of Otago, Dunedin, New Zealand

sheila.skeaff@otago.ac.nz

Am J Clin Nutr 2009;90:1264-1271

Background: The effects of severe iodine deficiency during critical periods of brain development are well documented. Little is known about the consequences of milder forms of iodine deficiency on neurodevelopment.

Objective: The authors aimed to determine whether supplementing mildly iodine-deficient children with iodine improves cognition.

Methods: A randomized, placebo-controlled, double-blind trial was conducted in 184 children aged 10-13 years in Dunedin, New Zealand. Children were randomly assigned to receive a daily tablet containing either $150 \mu \mathrm{g}$ iodine or placebo for 28 weeks. Biochemical, anthropometric, and dietary data were collected from each child at baseline and after 28 weeks. Cognitive performance was assessed through 4 subtests from the Wechsler Intelligence Scale for Children.

Results: At baseline, children were mildly iodine-deficient (median urinary iodine concentration (UIC): $63 \mu \mathrm{g} / \mathrm{l}$; thyroglobulin concentration: $16.4 \mu \mathrm{g} / \mathrm{l})$. After 28 weeks, iodine status improved in the supplemented group (UIC: $145 \mu \mathrm{g} / \mathrm{l}$; thyroglobulin: $8.5 \mu \mathrm{g} / \mathrm{l}$ ), whereas the placebo group remained iodine- 
deficient (UIC: $81 \mu \mathrm{g} / \mathrm{l}$; thyroglobulin: $11.6 \mu \mathrm{g} / \mathrm{l}$ ). Iodine supplementation significantly improved scores for 2 of the 4 cognitive subtests (picture concepts $(\mathrm{p}=0.023)$ and matrix reasoning $(\mathrm{p}=0.040)$ ) but not for letter-number sequencing $(\mathrm{p}=0.480)$ or symbol search $(\mathrm{p}=0.608)$. The overall cognitive score of the iodine-supplemented group was 0.19 SD higher than that of the placebo group $(\mathrm{p}=0.011)$.

Conclusion: Iodine supplementation improved perceptual reasoning in mildly iodine-deficient children and suggests that mild iodine deficiency could prevent children from attaining their full intellectual potential.

The elimination of iodine deficiency by the year 2005 was a World Fit for Children target, yet a large proportion of children worldwide still have inadequate iodine intakes. Mild iodine deficiency remains the most common thyroid disease worldwide, and an important health issue also in industrialized countries. The impact of iodine supplementation on moderately iodine-deficient children was first shown in an elegant randomized controlled double-blind study by Zimmermann et al. [19]. lodine treatment significantly improved performance in 4 of 7 cognitive and motor tests.

The re-emergence of iodine deficiency in New Zealand is believed to be a consequence of lower concentrations of iodine in milk because of the discontinuation of iodine-containing sanitizers in the dairy industry, declining use of iodized salt, and an increased consumption of processed foods not made with iodized salt. Gordon et al. present us with convincing data on the impact of iodine supplementation of children in a mildly iodine-deficient area. They used a similar study design (duration of supplementation, cognitive testing) at a lower supplementation dose in analogy to the previous paper. The authors show significant association between iodine supplementation and improvement in 2 of 4 cognitive tests relative to placebo. The strength of this paper is to highlight the fact that not only in moderately but even in mildly iodine-deficient areas, iodine supplementation is beneficial for cognitive function in schoolchildren.

\section{Thyroid hormone resistance revisited New pathophysiological insights}

\section{Resistance to thyroid hormone is associated with raised energy expenditure, muscle mitochondrial uncoupling, and hyperphagia}

Mitchell CS, Savage DB, Dufour S, Schoenmakers N, Murgatroyd P, Befroy D, Halsall D, Northcott S, Raymond-Barker P, Curran S, Henning E, Keogh J, Owen P, Lazarus J, Rothman DL, Farooqi IS, Shulman GI, Chatterjee K, Petersen KF

University of Cambridge Metabolic Research Laboratories, Institute of Metabolic Science, Addenbrooke's Hospital, Cambridge, UK

kitt.petersen@yale.edu, kkc1@mole.bio.cam.ac.uk

J Clin Invest 2010;120:1345-1354

Background: Resistance to thyroid hormone (RTH), a dominantly inherited disorder usually associated with mutations in thyroid hormone receptor- $\beta$ (THRB), is characterized by elevated levels of circulating thyroid hormones (including thyroxine), failure of feedback suppression of thyrotropin, and variable tissue refractoriness to thyroid hormone action. Raised energy expenditure and hyperphagia are recognized features of hyperthyroidism, but the effects of comparable hyperthyroxinemia in RTH patients are unknown.

Results: The authors showed that resting energy expenditure (REE) was substantially increased in adults and children with THRB mutations. Energy intake in RTH subjects was increased by $40 \%$, with marked hyperphagia particularly evident in children. Rates of muscle TCA cycle flux were increased by $75 \%$ in adults with RTH, whereas rates of ATP synthesis were unchanged, as determined by ${ }^{13} \mathrm{C} /{ }^{31} \mathrm{P}$ magnetic resonance spectroscopy. Mitochondrial coupling index between ATP synthesis and mitochondrial rates of oxidation (as estimated by the ratio of ATP synthesis to TCA cycle flux) was significantly decreased in RTH patients.

Conclusion: These data demonstrate that basal mitochondrial substrate oxidation is increased and energy production in the form of ATP synthesis is decreased in the muscle of RTH patients and that resting 
oxidative phosphorylation is uncoupled in this disorder. Furthermore, these observations suggest that mitochondrial uncoupling in skeletal muscle is a major contributor to increased REE in patients with RTH, due to tissue selective retention of THRB sensitivity to elevated thyroid hormone levels.

This paper reports on extensive studies of energy balance in patients with thyroid hormone resistance (RTH), providing general insight into thyroid hormone-dependent energy metabolism in humans. RTH is characterized by a variable degree of tissue hyposensitivity to thyroid hormones usually associated with mutations in the thyroid hormone receptor- $\beta$ (THRB) [20]. The authors studied an unselected group of 54 adults and 13 children from 35 unrelated families harboring 25 different $T H R B$ gene mutations. First, the authors documented significantly increased resting energy expenditure and basal metabolic rate in patients with $\mathrm{RHT}$, using both ventilated hood and chamber calorimetry, respectively. The raised energy expenditure was positively correlated with heart rate and thyroid hormone levels being intermediate in RTH patients compared to euthyroid control and thyrotoxic subjects. Second, they analyzed food intake, measured by an ad libitum test meal and found a $75 \%$ increase in RTH children compared to controls, reaching levels present in hyperphagic patients with monogenetic obesity syndromes. Third, by investigating the role of skeletal muscle mitochondrial energy metabolism, they revealed a $75 \%$ increase in substrate oxidation but an identical level of ATP synthesis in the muscle of RHT patients compared to controls, suggesting that resting mitochondrial uncoupling in skeletal muscle is a major contributor to increased resting energy expenditure in patients with RTH. In conclusion, the authors suggest that thyroid hormone receptor- $\alpha$-rich organs such as skeletal muscle and myocardium are the main determinants of thyroid hormone-mediated changes in the whole body expenditure in humans.

\title{
New hope?
}

\section{A thyroid hormone analog with reduced dependence on the monocarboxylate transporter 8 for tissue transport}

\author{
Di Cosmo C, Liao XH, Dumitrescu AM, Weiss RE, Refetoff S \\ Department of Medicine, University of Chicago, Chicago, III., USA \\ refetoff@uchicago.edu \\ Endocrinology 2009;150:4450-4458
}

Background: Mutations of the thyroid hormone (TH) cell membrane transporter MCT8, on chromosome$\mathrm{X}$, produce severe mental and neurological impairment in men.

Methods: The authors generated a Mct8-deficient mouse (Mct8KO) manifesting the human thyroid phenotype. Although these mice have no neurological manifestations, they have decreased brain $\mathrm{T}_{3}$ content and high deiodinase 2 (D2) activity, reflecting TH deprivation. In contrast and as in serum, liver $\mathrm{T}_{3}$ content is high, resulting in increased deiodinase 1 (D1), suggesting that in this tissue TH entry is Mct8 independent. They tested the effect of 3,5-diiodothyropropionic acid (DITPA), a TH receptor agonist, for its dependence on Mct8 in Mct8KO and wild-type (Wt) mice tissues. After depletion of endogenous $\mathrm{TH}$, mice were given three different doses of DITPA. Effects were compared with treatment with two doses of $\mathrm{L}-\mathrm{T}_{4}$.

Results: As expected, physiological doses of $\mathrm{L}^{-\mathrm{T}_{4}}$ normalized serum TSH, brain D2, and liver D1 in Wt mice but not the Mct8KO mice. The higher dose of $\mathrm{T}_{4}$ suppressed TSH in the Wt mice, normalized TSH and brain D2 in Mct8KO mice, but produced a thyrotoxic effect on liver D1 in both genotypes. In contrast, DITPA produced similar effects on TSH, D2, and D1 in both Wt and Mct8KO mice. The higher dose fully normalized all measurements and other parameters of TH action.

Conclusions: DITPA is relatively MCT8 independent for entry into the brain and corrects the TH deficit in Mct8KO mice without causing thyrotoxic effect in liver. The potential clinical utility of this analog to patients with MCT8 mutations requires further studies.

The intracellular hypothyroidism in the brain due to the abolished activity of the thyroid hormone transporter MCT8 cannot be corrected by physiological or supraphysiological substitutive doses of 
thyroid hormones in patients with MTC8 mutations. In this context, the question, whether thyroid hormone analogues, such as the 3,5-diiodothyropropionic acid (DITPA), could be less dependent on the active transport by MTC8 for their passage from the blood to the central neurons, would be of great therapeutic importance.

Di Cosmo et al. show that the transport of DITPA into the brain and liver is less dependent on MTC8. Further, they provide evidence that DITPA acts in brain without producing thyrotoxic effects in peripheral tissues, such as the liver. Although, these results are an important step forward to a clinical study of this pharmacological compound in patients with MTC8 mutations, relevant species-specific differences have to be considered. As the MTC8 knockout mouse model does not display a neurological phenotype like MTC8-deficient humans, the potential effect on the severe human neurological phenotype remains to be proven.

\title{
New concerns?
}

\section{Glucagon-like peptide-1 receptor agonists activate rodent thyroid C-cells causing calcitonin release and C-cell proliferation}

\author{
Bjerre Knudsen L, Madsen LW, Andersen S, Almholt K, de Boer AS, Drucker DJ, Gotfredsen C, Egerod FL, \\ Hegelund AC, Jacobsen H, Jacobsen SD, Moses AC, Mølck AM, Nielsen HS, Nowak J, Solberg H, Thi TD, \\ Zdravkovic M \\ Department of Biology and Pharmacology Mgt, Novo Nordisk A/S, Novo Nordisk Park, Måløv, Denmark \\ lbkn@novonordisk.com \\ Endocrinology 2010;151:1473-1486
}

\begin{abstract}
Background: Liraglutide is a glucagon-like peptide-1 (GLP-1) analog developed for type 2 diabetes. Longterm liraglutide exposure in rodents was associated with thyroid C-cell hyperplasia and tumors.

Results: The GLP-1 receptor was localized to rodent C-cells. GLP-1 receptor agonists stimulated calcitonin release, up-regulation of calcitonin gene expression, and subsequently C-cell hyperplasia in rats and, to a lesser extent, in mice. In contrast, humans and/or cynomolgus monkeys had low GLP-1 receptor expression in thyroid C-cells, and GLP-1 receptor agonists did not activate adenylate cyclase or generate calcitonin release in primates. Moreover, 20 months of liraglutide treatment (at $>60$ times human exposure levels) did not lead to C-cell hyperplasia in monkeys. Mean calcitonin levels in patients exposed to liraglutide for 2 years remained at the lower end of the normal range, and there was no difference in the proportion of patients with calcitonin levels increasing above the clinically relevant cutoff level of $20 \mathrm{pg} / \mathrm{ml}$.

Conclusion: The findings delineate important species-specific differences in GLP-1 receptor expression and action in the thyroid. Indeed the data support a GLP-1 receptor-mediated mechanism for these changes in rodents. Nevertheless, the long-term consequences of sustained GLP-1 receptor activation in the human thyroid remain unknown and merit further investigation.
\end{abstract}

Glucagon-like peptide-1 (GLP-1) is an incretin hormone that promotes glucose-dependent stimulation of insulin secretion. GLP-1 receptor agonists with prolonged half-life have been developed for the treatment of type 2 diabetes. The FDA approved liraglutide for treatment of type 2 diabetes in January 2010. One major safety concern was the potential deleterious effect of liraglutide on thyroid C-cells, as the long-term exposure was associated with thyroid C-cell hyperplasia and tumors in rodents.

In this context, the authors combined short- and long-term animal and human pharmacological studies to show relevant species-dependent differences of GLP-1 receptor expression as well as calcitonin release to liraglutide between rodents (rat and mouse) and humans and primates. Two-year highdose exposure studies confirmed C-cell hyperplasia and development of C-cell adenoma in rodents. In contrast, no C-cell hyperplasia was observed in primates during and after 87 weeks of treatment and basal plasma calcitonin levels were not increased in patients with type 2 diabetes receiving liraglutide in three different doses compared to placebo at repeated measurements during 2 years. In conclusion, thyroid C-cells in rats and mice differ markedly from primate and human C-cells in their response 
to GLP-1 receptor activation supporting the arguments of the FDA for approval of the drug. Longterm safety monitoring is nevertheless required by the FDA by monitoring the annual incidence of medullary thyroid cancer of over the next 15 years. For the complete discussion of the safety issue of liraglutide by the FDA, the interested reader is referred to the article by Parks and Rosebraugh [21].

References

1. Rivkees SA, Mattison DR: Ending propylthiouracil-induced liver failure in children. N Engl J Med 2009;360:15741575 .

2. Bahn RS, Burch HS, Cooper DS, et al: The role of propylthiouracil in the management of Graves' disease in adults: report of a meeting jointly sponsored by the American Thyroid Association and the Food and Drug Administration. Thyroid 2009;19:673-674.

3. Clementi M, Di Gianantonio E, Pelo E, Mammi I, Basile RT, Tenconi R: Methimazole embryopathy: delineation of the phenotype. Am J Med Genet 1999;83:43-46.

4. Karlsson FA, Axelsson O, Melhus H: Severe embryopathy and exposure to methimazole in early pregnancy. J Clin Endocrinol Metab 2002;87:947-949.

5. Valdez RM, Barbero PM, Liascovich RC, De Rosa LF, Aguirre MA, Alba LG: Methimazole embryopathy: a contribution to defining the phenotype. Reprod Toxicol 2007;23:253-255.

6. Luton D, Le Gac I, Vuillard E, et al: Management of Graves' disease during pregnancy: the key role of fetal thyroid gland monitoring. J Clin Endocrinol Metab 2005;90:6093-6098.

7. Van Vliet G, Polak M, Ritzen EM: Treating fetal thyroid and adrenal disorders through the mother. Nat Clin Pract Endocrinol Metab 2008;4:675-682.

8. Polak M, Van Vliet G: Therapeutic approach of fetal thyroid disorders. Horm Res Paediatr 2010;74:1-5.

9. Baloch Z, Carayon P, Conte-Devolx B, et al: Laboratory medicine practice guidelines. Laboratory support for the diagnosis and monitoring of thyroid disease. Thyroid 2003;13:3-126.

10. Hanna CE, Krainz PL, Skeels MR, Miyahira RS, Sesser DE, LaFranchi SH: Detection of congenital hypopituitary hypothyroidism: ten-year experience in the Northwest Regional Screening Program. J Pediatr 1986;109:959-964.

11. Asakura Y, Tachibana K, Adachi M, Suwa S, Yamagami Y: Hypothalamo-pituitary hypothyroidism detected by neonatal screening for congenital hypothyroidism using measurement of thyroid-stimulating hormone and thyroxine. Acta Paediatr 2002;91:172-177.

12. Schlumberger M, Ricard M, De Pouvourville G, Pacini F: How the availability of recombinant human TSH has changed the management of patients who have thyroid cancer. Nat Clin Pract Endocrinol Metab 2007;3:641-650.

13. Krude H, Schutz B, Biebermann H, et al: Choreoathetosis, hypothyroidism, and pulmonary alterations due to human NKX2-1 haploinsufficiency. J Clin Invest 2002;109:475-480.

14. Moreno JC, Bikker H, Kempers MJ, et al: Inactivating mutations in the gene for thyroid oxidase 2 (THOX2) and congenital hypothyroidism. N Engl J Med 2002;347:95-102.

15. Ku CS, Loy EY, Salim A, Pawitan Y, Chia KS: The discovery of human genetic variations and their use as disease markers: past, present and future. J Hum Genet 2010 (in press).

16. Manolio TA, Brooks LD, Collins FS: A HapMap harvest of insights into the genetics of common disease. J Clin Invest 2008;118:1590-1605.

17. Zhang F, Gu W, Hurles ME, Lupski JR: Copy number variation in human health, disease, and evolution. Annu Rev Genomics Hum Genet 2009;10:451-481.

18. Craddock N, Hurles ME, Cardin N, et al: Genome-wide association study of CNVs in 16,000 cases of eight common diseases and 3,000 shared controls. Nature 2010;464:713-720.

19. Zimmermann MB, Connolly K, Bozo M, Bridson J, Rohner F, Grimci L: Iodine supplementation improves cognition in iodine-deficient schoolchildren in Albania: a randomized, controlled, double-blind study. Am J Clin Nutr 2006;83:108114.

20. Weiss RE, Refetoff S: Resistance to thyroid hormone. Rev Endocr Metab Disord 2000;1:97-108.

21. Parks M, Rosebraugh C: Weighing risks and benefits of liraglutide - the FDA's review of a new antidiabetic therapy. N Engl J Med 2010;362:774-777. 